\title{
BMJ Open Impact of tailored falls prevention education for older adults at hospital discharge on engagement in falls prevention strategies postdischarge: protocol for a process evaluation
}

\author{
Chiara Naseri, ${ }^{1}$ Steven M McPhail, ${ }^{2,3}$ Julie Netto, ${ }^{4}$ Terrence $\mathrm{P}$ Haines, ${ }^{5}$ \\ Meg E Morris, ${ }^{6}$ Christopher Etherton-Beer, ${ }^{7}$ Leon Flicker, ${ }^{8}$ Den-Ching A Lee, ${ }^{5}$ \\ Jacqueline Francis-Coad, ${ }^{9}$ Anne-Marie Hill ${ }^{1}$
}

To cite: Naseri C, McPhail SM, Netto J, et al. Impact of tailored falls prevention education for older adults at hospital discharge on engagement in falls prevention strategies postdischarge: protocol for a process evaluation. BMJ Open 2018;8:e020726. doi:10.1136/ bmjopen-2017-020726

- Prepublication history for this paper is available online. To view these files, please visit the journal online (http://dx.doi. org/10.1136/bmjopen-2017020726).

Received 20 November 2017 Revised 28 February 2018 Accepted 7 March 2018

Check for updates

For numbered affiliations see end of article.

Correspondence to Chiara Naseri;

chiara.naseri@postgrad.curtin. edu.au

\section{ABSTRACT}

Introduction Older adults recently discharged from hospital have greater incidence of adverse events, functional decline, falls and subsequent readmission. Providing education to hospitalised patients on how to prevent falls at home could reduce postdischarge falls. There has been limited research investigating how older adults respond to tailored falls prevention education provided at hospital discharge. The aim of this study is to evaluate how providing tailored falls prevention education to older patients at the point of, and immediately after hospital discharge in addition to usual care, affects engagement in falls prevention strategies in the 6-months postdischarge period, including their capability and motivation to engage in falls prevention strategies. Methods and analyses This prospective observational cohort study is a process evaluation of a randomised controlled trial, using an embedded mixed-method design. Participants $(n=390)$ who have been enrolled in the trial are over the age of 60 years, scoring greater than $7 / 10$ on the Abbreviated Mental Test Score. Participants are being discharged from hospital rehabilitation wards in Perth, Western Australia, and followed up for 6 months postdischarge. Primary outcome measures for the process evaluation are engagement in falls prevention strategies, including exercise, home modifications and receiving assistance with activities of daily living. Secondary outcomes will measure capability, motivation and opportunity to engage in falls prevention strategies, based on the constructs of the Capability Opportunity Motivation Behaviour system. Quantitative data are collected at baseline, then at 6 months postdischarge using structured phone interviews. Qualitative data are collected from a purposive sample of the cohort, using semistructured in-depth phone interviews. Quantitative data will be analysed using regression modelling and qualitative data will be analysed using interpretive phenomenological analysis. Ethics and dissemination Results will be presented in peer-reviewed journals and at conferences worldwide. This study is approved by hospital and university Human Research Ethics Committees.
Strengths and limitations of this study

- This is a process evaluation of a randomised controlled trial (RCT) representing a broad cohort of older adults recruited from three public metropolitan rehabilitation hospitals in Australia.

- The education intervention delivered in the RCT is being evaluated for its effect on falls rates following hospital discharge. This process evaluation will assess participants' response to the education, which aims to increase older adults' engagement in falls prevention strategies after they are discharged from hospital.

- It will determine if providing falls prevention education can facilitate capability, opportunity and motivation for older adults to engage in falls prevention strategies at home after hospital discharge.

- The prospective design, robust data collection and the convergent embedded mixed-method design uses triangulation to describe the effects of the education on engagement in falls prevention strategies, to outline barriers to engagement and provide a more holistic understanding of the factors that mediate the effectiveness of the education.

- A possible limitation is that the participants have been drawn from a high-risk population that may still be affected by their illness

\section{INTRODUCTION}

Globally, falls and falls-related injuries have been identified as a major public health problem associated with population ageing, causing physical injuries including hip fracture, head injury and negatively impacting quality of life among older people. ${ }^{1-3}$ In 2015, direct medical costs for fatal falls in the USA have more than tripled since the year $2000,{ }^{4}$ and in Australia the age-standardised falls-related hospitalisations for older adults has continued to increase by $2.3 \%$ per year. ${ }^{5}$ 
Direct costs do not account for the long-term effects of these injuries such as permanent disability, dependence on others and reduced participation in life. ${ }^{67}$

Falls are known to be increased among older adults who have been discharged from hospital, ${ }^{89}$ and it is also known that hospitalisation of older adults, including those who are admitted for acute care and rehabilitation, is associated with decline in function and mobility. ${ }^{10} \mathrm{At}$ least $40 \%$ of older adults fall at least once in the 6-month period following hospital discharge, with more than half of falls resulting in an injury. ${ }^{12}{ }^{13}$ This is substantially higher than the annual rate of falls (30\%) and injurious falls $(10 \%)$ reported in the general community. ${ }^{14}$

There is evidence for the effectiveness of exercise and physical activity, ${ }^{15} 16$ along with home safety modifications and vitamin D supplementation, ${ }^{17}{ }^{18}$ in reducing falls among older community dwelling adults including those with comorbidities. However, this evidence does not specifically apply to the older postdischarge population. A wide variety of interventions have been evaluated for their efficacy in improving transitions from hospital to home, but these have not focused specifically on falls prevention, and reviews suggest they produce limited positive outcomes and do not significantly reduce adverse events including falls. ${ }^{19-21}$

Older people have been found to have low levels of awareness of their falls risks and the benefits of falls prevention strategies, despite their increased falls risk during the postdischarge period. ${ }^{22-24}$ A recent study showed that older people understood and effectively engaged in their discharge plan, yet experienced unanticipated problems, such as difficulty taking medications, uncontrolled pain, poor dietary intake and fragmented social supports, indicating that more support may be required. ${ }^{25}$ A pilot randomised controlled trial (RCT) demonstrated that tailored education was received positively by older adults and resulted in increased engagement in falls prevention strategies after discharge,${ }^{26}$ and a recent systematic review found that falls prevention programmes that contained a patient education component were effective in reducing rate of falls after hospital discharge. ${ }^{27}$ However, there have been no RCTs to date to show that using patient education alone can reduce falls after discharge. An $\mathrm{RCT}^{28}$ is the first trial being undertaken to evaluate whether providing tailored falls prevention education, that includes individual health professional consultations in hospital and after discharge in addition to usual care, reduces falls rates in older adults after discharge from hospital. The protocol for the RCT has been published previously. ${ }^{28}$

The education intervention has been developed using the framework of the Capability Opportunity Motivation Behaviour (COM-B) model of health behaviour change. ${ }^{29}$ The aim of the education is to increase engagement in falls prevention strategies, therefore it is important to understand the intended effect on this intermediate outcome of engagement in falls prevention strategies. It is yet to be determined if providing tailored falls prevention education can facilitate capability, opportunity and motivation for older adults to engage in falls prevention strategies at home after hospital discharge (figure 1).

\section{STUDY AIMS}

The primary aim is to evaluate the impact of tailored falls prevention education provided at hospital discharge in addition to usual care, on older adults' engagement in falls prevention strategies in the 6 months after hospital discharge. This will be compared with those who receive usual care alone. The secondary aims are (A) To evaluate older adults' capability, and motivation, to engage falls prevention strategies for those participants who received tailored falls prevention education in addition to usual care, compared with those that received a social/control intervention in addition to usual care. (B) To identify the opportunity (social and physical environment) surrounding the participant that made the behaviour possible, by exploring the barriers and facilitators identified by older adults to engage in falls prevention strategies in the 6 months following hospital discharge.

\section{METHOD \\ Design}

The study design comprises a process evaluation of an RCT currently being conducted in Perth, Australia. ${ }^{28}$ The protocol for the RCT has been previously published. ${ }^{28}$ This process evaluation uses a convergent embedded mixed method design; ${ }^{30}$ as both quantitative and qualitative data will be collected, analysed, then merged to enrich the interpretation of the results through methodological triangulation. Measuring engagement is a complex concept. ${ }^{31}$ By using triangulation to describe the effects of the education on engagement in falls prevention strategies through both quantitative and qualitative data sources, this aims to provide a more holistic understanding of the phenomena. ${ }^{32} 33$

\section{Ethical considerations}

Participant information forms are provided at the time of consent at baseline in hospital as a part of the RCT and all participants will provide written informed consent to participate in the study.

\section{Patient involvement}

Patients were not directly involved in the design of this process evaluation. Participants are informed at enrolment that they can elect to receive a plain language summary of results when the process evaluation is completed, each participant is reminded of this during the final phone call contact with researchers. Participants will be acknowledged and thanked for their contributions during the publication and distribution of results.

\section{Setting and participants}

The setting and participants for the RCT have been described in full previously. ${ }^{28}$ Briefly, participants $(n=390)$ 


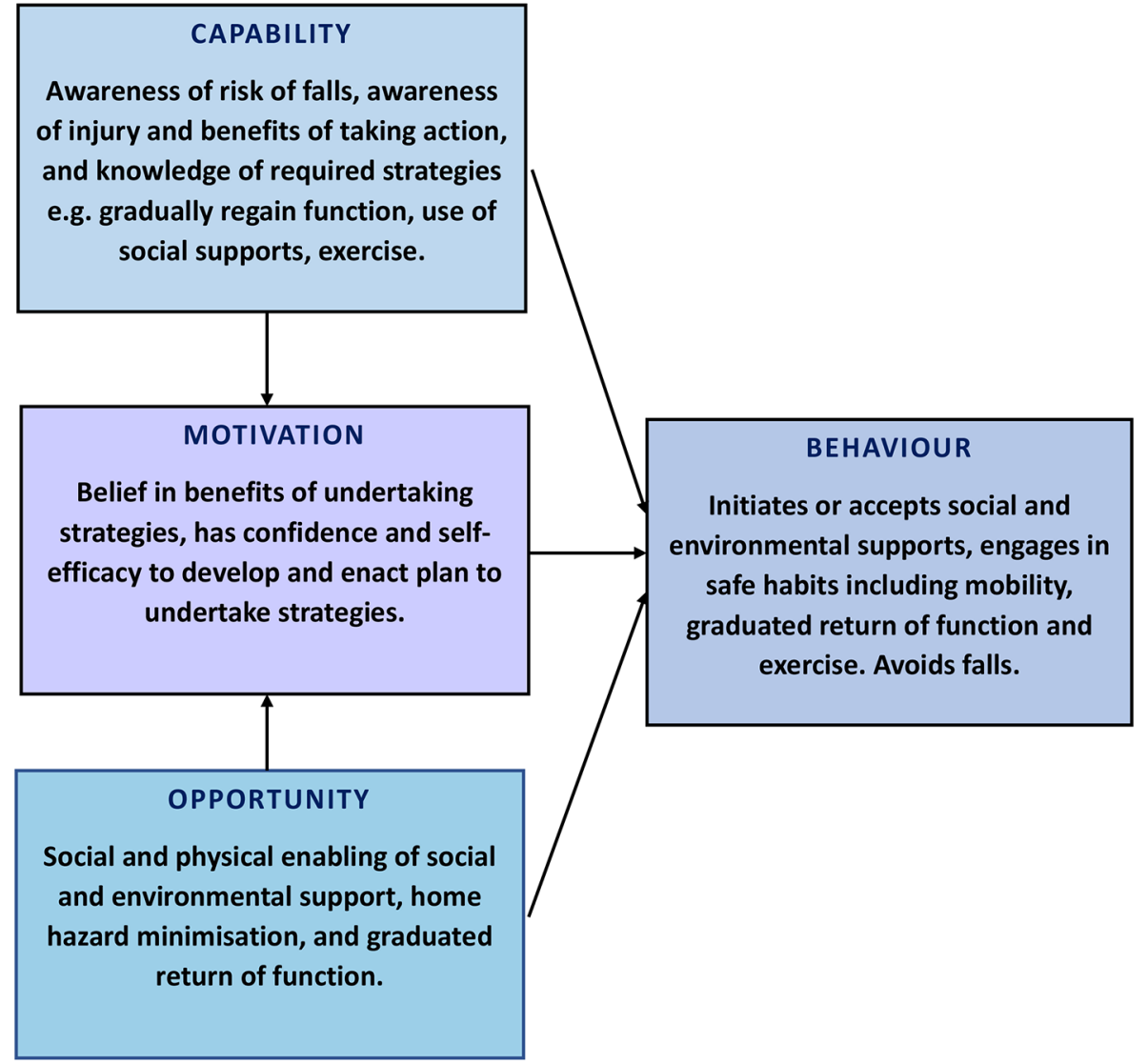

Figure 1 The Capability Opportunity Motivation Behaviour system applied to falls prevention behaviour postdischarge.

are recruited in hospital, ${ }^{28}$ provide written informed consent and are then randomly assigned (concealed) to either the intervention group or the control group prior to discharge from aged care rehabilitation and stroke units at three Western Australian hospitals. These wards admit patients with a variety of diagnoses, such as osteoarthritis, recent stroke, Parkinson's disease, dementia, recent orthopaedic or general surgery, or recovering from a general medical condition.

\section{Inclusion and exclusion criteria}

Inclusion criteria for the RCT have been described previously. ${ }^{28}$ All participants will contribute data for this process evaluation. Briefly, participants must be 60 years of age or older, and have cognitive function rated $>7 / 10$ in the Abbreviated Mental Test Score. ${ }^{34}$

\section{Education intervention}

The education intervention, which has been described in full previously, ${ }^{28}$ is based on a pedagogically sound programme found to be effective in improving knowledge, confidence and motivation for older patients to engage in falls prevention strategies after hospital discharge. ${ }^{26}$ The programme is planned to take between two and four sessions to deliver in an estimated total time of $45 \mathrm{~min}$. The education is delivered by physiotherapists and includes providing written and video materials followed by individualised discussion. The education content is based on the principles of health behaviour change, with messages that include falls prevention strategies tailored for each participant, such as instructions on how to engage in exercise according to their capability, to modify home hazards, to use their walking aid, to return to normal function, and how to seek assistance if required for home tasks or personal care. ${ }^{28}$

The control group receives a social intervention, between one and three sessions (estimated total time of $45 \mathrm{~min}$ ) with a trained health professional who discusses aspects of positive ageing using a scripted programme, without any falls prevention information.

The intervention is delivered in addition to usual inpatient care, including discharge planning, falls education, home-visits and equipment provision, and addition of social supports. ${ }^{28}$

\section{Outcome measures}

\section{Quantitative}

Primary outcome: engagement in falls prevention strategies in the 6 months after discharge. Falls prevention strategies measured are those suggested to the participant as a part of the tailored education intervention, which is based on current evidence for falls prevention, provided prior to discharge. Each participant has been encouraged to engage in a falls prevention plan which has been tailored by the delivering therapists. This intervention has been described in full elsewhere. ${ }^{28}$ Strategies are defined as: 
1. Receiving assistance (both formal and informal assistance) with activities of daily living (ADL). ADL are defined according to the Katz index of Independence in Activities of Daily Living, ${ }^{35}$ and include toileting, showering and eating.

2. Receiving assistance with instrumental ADL (IADL). IADL are defined using the Lawton Index, ${ }^{36}$ and include home cleaning, shopping and transport.

These two outcomes will be measured using yes/no responses and frequency (days per week and hours of total assistance per week) and type of assistance (whether paid formal services from home care provider or informal family or friends' assistance to the participant).

3. Engagement in exercises, including all types (such as a strength and balance exercise programme, group exercise, swimming, golf, tai chi, walking, dancing), whether a balance component is included, and frequency (hours per week and number of times per week) and where completed such as at home, in a healthcare centre, with or without health provider assistance.

4. Home modifications, such as installation of equipment or rails, or alteration of home layout, including whether assessment was provided by an occupational therapist and the level of assistance obtained to make these modifications.

These primary outcomes will be measured in hospital (baseline) by recruiters for the RCT (who are blinded to group allocation), then measured at 6 months following hospital discharge through a structured phone survey by a trained research assistant who is also blinded to group allocation. These surveys have been modified from previous surveys used in falls prevention trials, including the pilot trial which evaluated these outcomes. ${ }^{132637}$

The secondary quantitative outcome measures are:

1. Participants perceived levels of capability (knowledge and awareness) about falls prevention after discharge, such as awareness of risk of falls, awareness of injury and benefits of engaging falls prevention strategies; measured through a structured phone survey using Likert Scales, ${ }^{38}$ at baseline and at 6 months follow-up.

2. Motivation, such as beliefs in benefits of engaging strategies, confidence to engage strategies; develop and enact plans to engage strategies.

These secondary outcomes will be measured alongside the primary outcomes, using the methods described above. Survey items for secondary outcomes will be measured using 5-point Likert Scales, ${ }^{38}$ (strongly agree to strongly disagree). Items are based on the domains of COM-B,${ }^{29}$ and modified from previous surveys which have evaluated capability, motivation and confidence regarding falls prevention. ${ }^{13} 2637$

3. Motivation to engage in exercise will be additionally measured using the Self-Efficacy for Exercise Scale (SEE). ${ }^{39}$

The $\mathrm{SEE}^{39}$ is a nine items scale that rates older peoples' response to a statement about barriers to exercise (scores range from $0=$ notvery confident to $10=$ very confident; with a total possible score of 90).

\section{Qualitative}

The secondary qualitative outcomes relate to opportunity (described as being both social and physical in the COM-B framework), ${ }^{29}$ and include both barriers and enablers that participants encounter when seeking to engage in falls prevention strategies. These secondary outcomes will be measured by completing semistructured in-depth phone interviews at the conclusion of the observation period. Questions will be guided by participant responses gained from earlier structured phone interviews, using openended questions designed to encourage the participants to reflect on their previous responses. Questions will be framed around barriers and enablers to engaging in falls prevention strategies, graduated return to independence and engaging in exercise. This may be physical opportunity provided by their environment including access and social supports, or cultural milieu including stigmas or fears that dictate older adult decision-making. ${ }^{29}$

Demographic data will be gathered in hospital at baseline by recruiters during a face-to-face interview. These data will include age, gender, diagnosis, length of stay in hospital, history of falls prior to hospitalisation and during hospital stay, presence of visual impairment, presence of hearing impairment, number and type of medications, signs of depression (measured using the Geriatric Depression Scale), ${ }^{40}$ and use of walking aids.

Other data are also collected at baseline during the faceto-face interview then again at 6 months after discharge using a structured phone survey. These variables are living situation (home alone, with partner, other situation), level of indoor and outdoor mobility, including any use of walking aids, functional mobility measured using Katz and Lawton's scales, ${ }^{536}$ and health-related quality of life measured using the Assessment of Quality of Life tool. ${ }^{41}$

Additionally, as part of the education intervention, data are collected regarding the delivery of the programme by the educators. These data include the number of education sessions provided to each intervention group participant, the duration and whether an action plan was completed. These data will also be used during sensitivity analyses, to assist to explain participants' knowledge, motivation and engagement in falls prevention strategies after discharge.

\section{Data collection and procedure}

Baseline surveys for primary and secondary outcomes are conducted by a trained research assistant who is blinded to group allocation, then participants are randomly allocated to intervention or control group. The RCT protocol, including randomisation, blinding and the intervention procedure has been described in detail elsewhere.$^{28}$ Briefly, participants receive tailored falls prevention education by trained physiotherapist educators during a one-to-one interaction in hospital. The education assists the participant to prepare a tailored plan to 
initiate after hospital discharge. The participants are then followed up by phone after discharge by the educators once a month for 3 months, to further assist them to enact their plan, and address any barriers that may have arisen since discharge.

At 6 months following hospital discharge, the structured phone survey will be conducted to collect quantitative follow-up data, after which the participant will be invited to participate in a semistructured in-depth phone interview to collect qualitative data that measure the secondary outcome which explores opportunity (barriers and enablers) to engagement in falls prevention strategies.

Purposive sampling for qualitative data collection will occur after the 6-month period and following completion of primary and secondary quantitative data collection. The sample selected will represent the cohort, with consideration of age, diagnosis, gender, falls history, and whether intervention or control group. Purposive sampling will be finalised and justified by referring to data and theoretical saturation and confirmed through consensus of a second researcher reviewing the transcribed narrative data. ${ }^{42} \mathrm{~A}$ phone interview was selected to collect data, rather than a focus group, or face-to-face interview, as the participants have previously received monthly phone monitoring of falls data from the RCT, so the researcher has established a genuine rapport and reciprocity with the participants. ${ }^{43}$ To ensure quality data collection that is sufficient to answer the study aim, the semistructured survey has been piloted to ensure the questions are easily understood and screened for blind spots, bias and potentially sensitive questions. ${ }^{33}$ Each interview will be recorded and transcribed verbatim. Additional interviews will be completed as necessary until data saturation has occurred. The researcher will keep a journal to record observations and reflections regarding data collection and procedure. ${ }^{43}$

\section{Statistical analysis}

\section{Quantitative data}

Quantitative data will be analysed using Stata (Stata Statistical Software, StataCorp, College Station, Texas ,USA) ${ }^{44}$ and intention-to-treat analysis will be undertaken when examining potential influence of group allocation on process outcomes based on the trial randomisation. ${ }^{45}$ Primary and secondary outcomes will be summarised using descriptive statistics. The primary analysis will compare engagement with each strategy between the control and intervention groups for 6 months postdischarge from hospital, using regression models that will control for baseline measures of engagement and be conducted with adjustment for potential covariates consistent with the prior pilot study for this trial. ${ }^{26}$ Similarly, secondary analyses will compare the secondary outcomes to examine potential between-group differences using regression models that will control for baseline and be conducted with adjustment for potential covariates consistent with the prior pilot study for this trial. ${ }^{26}$ Sensitivity analyses will also be conducted to examine whether the trial findings are robust to planned analysis choices (eg, intention-to-treat versus as-treated analyses, or adjusted versus unadjusted regression models). The significance level for analyses will be set at 0.05 , and the sample size was determined by primary trial effect analysis, which has previously been described. ${ }^{28}$

\section{Qualitative data}

Qualitative data from researcher field notes, phone interview transcriptions and participant open-ended answers to structured questions in the quantitative survey will be used, with the intent to triangulate the different data sources and gain a multilayered understanding of the findings. ${ }^{3233}$ Interpretive phenomenological analysis (IPA) will be used to describe and interpret participants' behaviours regarding engagement in falls prevention strategies. ${ }^{46}$ Briefly, following IPA guidelines the two researchers will independently produce detailed interpretive coding of how and why the participants experienced barriers or enablers to engaging falls prevention strategies since hospital discharge. These coded data will then be examined by the two researchers together to identify emergent themes then re-examined to ascertain if it described the data collected and if all coded data were captured within these identified emergent themes. ${ }^{46}$ Member checking will occur by the first researcher returning to a sample of participants to ask them how accurately their realities have been represented in the final interpretations. ${ }^{42}$ To add rigour, a third researcher who is not involved in data collection, will then be invited to scrutinise the data and to arbitrate any differences between coding and themes, and review final interpretations. ${ }^{46}$ Purposive sampling for qualitative data collection will be finalised and justified by consensus between all three researchers referring to the findings to confirm saturation of themes. ${ }^{46}$

Finally, quantitative and qualitative data will be synthesised to enrich the interpretation of the findings with the aim of adding validity to the study. ${ }^{33} 42$ An overview of the procedure for primary and secondary quantitative and qualitative data collection and statistical analysis is presented in figure 2.

\section{DISCUSSION}

Older people are known to have increased rates of falls and functional decline following hospital discharge. ${ }^{78}$ Recent studies investigating readmissions have found that patients are unprepared to manage their physical limitations during their immediate recovery after hospital discharge. ${ }^{24} 25$ These investigations have shifted from a hospital-centric model to a patient-centred approach to understand the lived experience of older adults as they transition from hospital to home. ${ }^{47}$ This is important because other systematic reviews of discharge planning have identified that while readmissions may be reduced with such interventions, the impact on health outcomes for the patients is uncertain. ${ }^{48}$ 


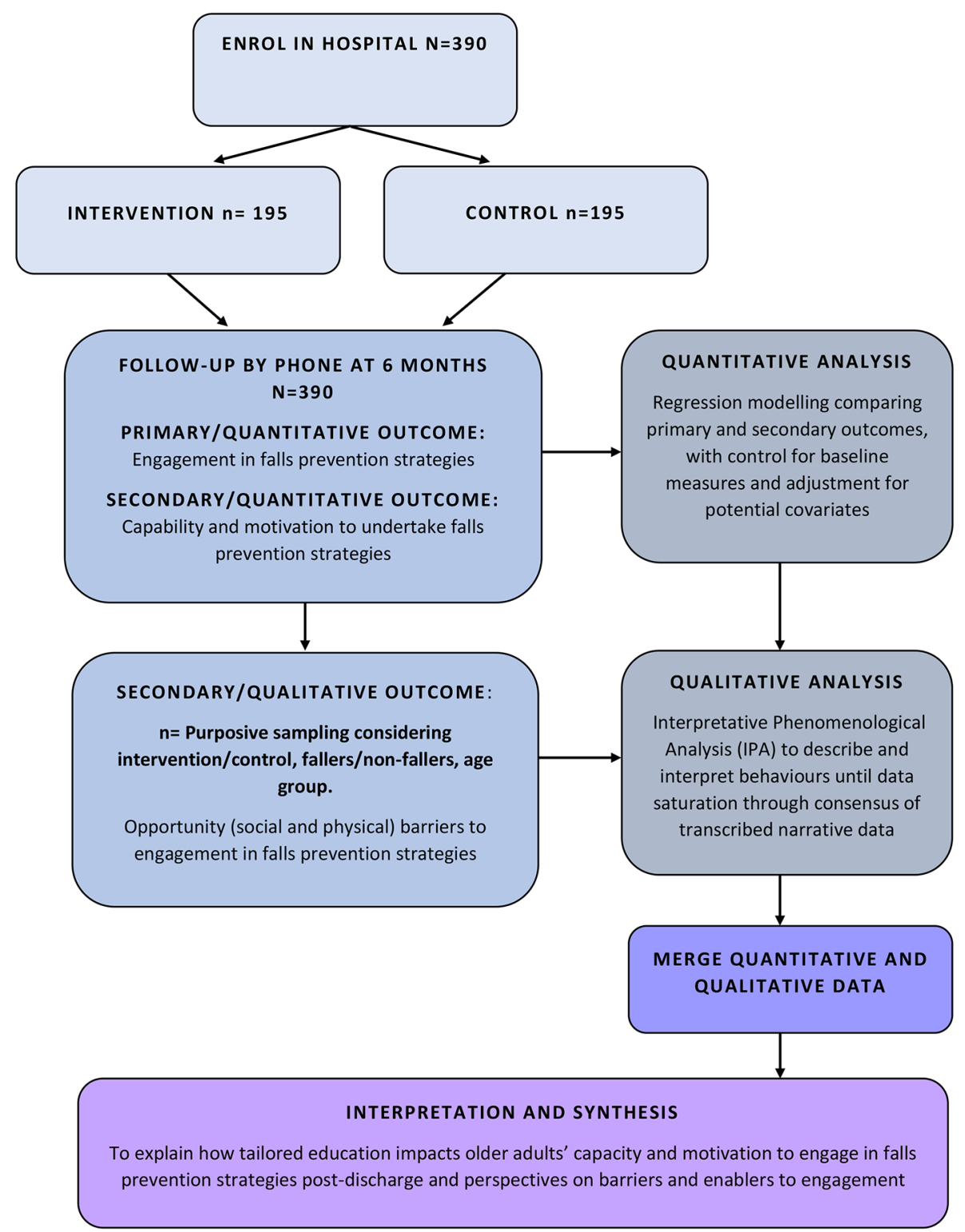

Figure 2 Study procedure

Previous observational studies have suggested that to promote participation in evidenced-based falls prevention strategies, therapists may need to convince older adults that they are at risk of falls, ${ }^{23}$ with guidance on what specific strategies are likely to have a personally beneficial falls prevention effect. ${ }^{22}$ Tailored health education aims to change individuals' health behaviours. ${ }^{29}{ }^{30}$ When this education is used as an intervention, it presents a challenge for identifying effective components, and therefore reporting of findings, and subsequent replication. ${ }^{31} 32$

This process evaluation will seek to understand whether providing tailored education facilitates older adults' engagement in falls prevention strategies following hospital discharge. The application of the framework of the COM-B model to the findings ${ }^{29}$ will assist to characterise how the intervention altered motivation, capability or opportunity. Additionally, secondary analysis of barriers or enablers to engagement will be mapped onto the COM-B model and subsequently identify more precise determinants of engagement. ${ }^{49}$ Capability includes an individual's psychological and physical capacity to engage in falls prevention strategies behaviour. Opportunity, both social and physical, includes those factors that lie outside the individual that make the behaviour possible, such as being able to access home assistance or modifications. ${ }^{29}$ Motivation includes all processes that inspire and direct behaviour, such as believing that it would be good to exercise. ${ }^{29}$

This study has strengths and limitations that warrant consideration. A strength is that the participants are a broad cohort recruited from a representative sample of three public metropolitan rehabilitation hospitals in Australia. The delivery of a falls prevention education intervention just prior to discharge with follow-up sessions by telephone during 1 month after hospital discharge has previously shown promising effects on older adult 
engagement in falls prevention strategies in a pilot trial. ${ }^{26}$ Other strengths include the prospective design, robust data collection and the convergent embedded mixedmethod design, which combines the advantages of both quantitative and qualitative data. ${ }^{32} 43$

A possible limitation is that the participants have been drawn from a high-risk population that may still be affected by their illness. To minimise bias through possible prompting of participants, data regarding engagement in falls prevention strategies following hospital discharge, will not be collected until 6 months postdischarge. We are also relying on self-reported data at 6 months. Participants are only contacted by phone and not interviewed face to face, however we have found in our earlier trials ${ }^{13,26}$ that this allows more complete responses as older people, especially if unwell, are not always able to attend a clinic setting.

\section{CONCLUSION}

This process evaluation will assess older adults' response to a tailored falls prevention education programme and investigate how the intervention was received and interpreted by the older participant during their postdischarge recovery. When delivering interventions that seek to facilitate health behaviour change, it is also important to understand the process by which behaviour changes and the mediating factors. ${ }^{50} 51$ This provides evidence to develop a sound basis for defining effective intervention components. ${ }^{52}$ We will clarify whether providing tailored falls prevention education can positively change health behaviour. We will also explore older adults' knowledge of falls prevention strategies and motivation to engage falls prevention strategies following hospital discharge. Findings will enable generation of robust recommendations for clinicians and researchers about the role of tailored falls prevention education at the point of hospital discharge. Ultimately, we aim to understand if providing older adults with tailored education enables them to change their health behaviour in the postdischarge period and if engagement in relevant strategies reduces falls after hospital discharge.

\section{Author affiliations}

${ }^{1}$ School of Physiotherapy and Exercise Sciences, Faculty of Health Science, Curtin University, Perth, Western Australia, Australia

${ }^{2}$ School of Public Health and Social Work, Faculty of Health, Queensland University of Technology, Brisbane, Queensland, Australia

${ }^{3}$ Centre for Functioning and Health Research, Metro South Health, Woolloongabba, Queensland, Australia

${ }^{4}$ School of Occupational Therapy and Social Work, Faculty of Health Science, Curtin University, Perth, Western Australia, Australia

${ }^{5}$ Faculty of Medicine, Nursing and Health Science, Monash University, Melbourne, Victoria, Australia

${ }^{6}$ Healthscope \& La Trobe Centre for Sport \& Exercise Medicine Research, La Trobe University, Melbourne, Victoria, Australia

${ }^{7}$ Department of Geriatric Medicine, School of Medicine and Pharmacology, Royal Perth Hospital Unit, University of Western Australia, Perth, Western Australia, Australia

${ }^{8}$ Western Australian Centre for Health \& Ageing, Centre for Medical Research, University of Western Australia, Perth, Western Australia, Australia
${ }^{9}$ School of Physiotherapy, Institute for Health Research, The University of Notre Dame Australia, Fremantle, Western Australia, Australia

Contributors A-MH, CN, SMM and TPH conceptualised the current study design and resultant research protocol with ongoing expertise and support from JN, CE-B, MEM and JF-C. A-MH and CN led trial management including data collection and management and site procedure, in consultation with TPH, MEM, CE-B and LF. A-MH, CN and SMM led statistical analyses with support from TPH, JN, D-CAL and JF-C. CN led the drafting of all sections of the manuscript in consultation with A-MH, SMM, JN, CE-B, MEM, LF and D-CAL. All authors critically revised the manuscript for important intellectual content and read and approved of the final version of the manuscript.

Funding Authors SMM, TPH, MEM, JF-C, CE-B, D-CAL, LF, A-MH have received a grant from the National Health and Medical Research Council (Australia) to conduct a trial which investigates how to reduce falls and promote independence in older people after hospital discharge (Project App no:1078918). CN receives a postgraduate student stipend as part of the grant. This grant funding has not been received directly to the authors rather to the institutions they represent. The authors have not received financial support for this study. SMM receives career funding support from the National Health and Medical Research Council (Project App no:1090440, Australia).

\section{Competing interests None declared.}

Patient consent Detail has been removed from this case description/these case descriptions to ensure anonymity. The editors and reviewers have seen the detailed information available and are satisfied that the information backs up the case the authors are making.

Ethics approval Ethics approvals have been obtained from Human Research Ethics Committee of North Metropolitan Health Service and South Metropolitan Health Service with reciprocal approval from The University of Notre Dame Australia and Curtin University.

Provenance and peer review Not commissioned; externally peer reviewed.

Open Access This is an Open Access article distributed in accordance with the Creative Commons Attribution Non Commercial (CC BY-NC 4.0) license, which permits others to distribute, remix, adapt, build upon this work non-commercially, and license their derivative works on different terms, provided the original work is properly cited and the use is non-commercial. See: http://creativecommons.org/ licenses/by-nc/4.0/

(c) Article author(s) (or their employer(s) unless otherwise stated in the text of the article) 2018. All rights reserved. No commercial use is permitted unless otherwise expressly granted.

\section{REFERENCES}

1. WHO. Falls - WHO Fact sheet $\mathrm{N}^{\circ} 344$. https://www.who.int/ mediacentre/factsheets/fs344/en/2012 (accessed 3 Mar 2016).

2. Hartholt KA, Van Lieshout EM, Polinder S, et al. Rapid increase in hospitalizations resulting from fall-related traumatic head injury in older adults in The Netherlands 1986-2008. J Neurotrauma 2011;28:739-44.

3. Wolinsky FD, Bentler SE, Liu L, et al. Recent hospitalization and the risk of hip fracture among older Americans. J Gerontol A Biol Sci Med Sci 2009;64:249-55.

4. Burns ER, Stevens JA, Lee R. The direct costs of fatal and non-fatal falls among older adults - United States. J Safety Res 2016;58:99-103.

5. Bradley C. AlHW. Trends in hospitalisations due to falls by older people, Australia 1999-00 to 2010-11. Injury research and statistics series 84. Cat. no. INJCAT 160. Canberra: AlHW, 2013.

6. Heinrich S, Rapp K, Rissmann U, et al. Cost of falls in old age: a systematic review. Osteoporos Int 2010;21:891-902.

7. Stevens JA, Corso PS, Finkelstein EA, et al. The costs of fatal and non-fatal falls among older adults. Inj Prev 2006;12:290-5.

8. Mahoney JE. Falls in the post-hospitalization period. Clin Geriatr 2005;13:39-46.

9. Davenport RD, Vaidean GD, Jones CB, et al. Falls following discharge after an in-hospital fall. BMC Geriatr 2009;9:53.

10. Heyland DK, Garland A, Bagshaw SM, et al. Recovery after critical illness in patients aged 80 years or older: a multi-center prospective observational cohort study. Intensive Care Med 2015;41:1911-20. 
11. Pritchard E, Warren N, Barker A, et al. Personal life approach: an interactive way of understanding older adults' participation in activities following hospitalization. Gerontologist 2016;56:504-13

12. Hill AM, Hoffmann T, Haines TP. Circumstances of falls and fallsrelated injuries in a cohort of older patients following hospital discharge. Clin Interv Aging 2013;8:765-74.

13. Hill AM, Hoffmann T, McPhail S, et al. Evaluation of the sustained effect of inpatient falls prevention education and predictors of falls after hospital discharge--follow-up to a randomized controlled trial. $J$ Gerontol A Biol Sci Med Sci 2011;66:1001-12.

14. Milat AJ, Watson WL, Monger C, et al. Prevalence, circumstances and consequences of falls among community-dwelling older people: results of the 2009 NSW Falls Prevention Baseline Survey. N S W Public Health Bull 2011;22:43-8.

15. Morris ME, Menz HB, McGinley JL, et al. A randomized controlled trial to reduce falls in people with parkinson's disease. Neurorehabil Neural Repair 2015;29:777-85.

16. Sherrington C, Tiedemann A, Fairhall N, et al. Exercise to prevent falls in older adults: an updated meta-analysis and best practice recommendations. N S W Public Health Bull 2011;22:78-83.

17. Gillespie LD, Robertson MC, Gillespie WJ, et al. Interventions for preventing falls in older people living in the community. Cochrane Database Syst Rev 2012;9:Cd007146.

18. Cumming RG, Thomas M, Szonyi G, et al. Home visits by an occupational therapist for assessment and modification of environmental hazards: a randomized trial of falls prevention. J Am Geriatr Soc 1999;47:1397-402.

19. Batchelor F, Hill K, Mackintosh S, et al. What works in falls prevention after stroke?: a systematic review and meta-analysis. Stroke 2010;41:1715-22.

20. Conroy SP, Stevens T, Parker SG, et al. A systematic review of comprehensive geriatric assessment to improve outcomes for frail older people being rapidly discharged from acute hospital: 'interface geriatrics'. Age Ageing 2011;40:436-43.

21. Shepperd S, Lannin NA, Clemson LM, et al. Discharge planning from hospital to home. Cochrane Database Syst Rev 2013:1:CD000313.

22. Mihaljcic T, Haines TP, Ponsford JL, et al. Investigating the relationship between reduced self-awareness of falls risk, rehabilitation engagement and falls in older adults. Arch Gerontol Geriatr 2017;69:38-44.

23. Haines TP, Day L, Hill KD, et al. "Better for others than for me": a belief that should shape our efforts to promote participation in falls prevention strategies. Arch Gerontol Geriatr 2014;59:136-44.

24. Hill AM, Hoffmann T, Beer C, et al. Falls after discharge from hospital: is there a gap between older peoples' knowledge about falls prevention strategies and the research evidence? Gerontologist 2011:51:653-62.

25. Greysen SR, Harrison JD, Kripalani S, et al. Understanding patientcentred readmission factors: a multi-site, mixed-methods study. BMJ Qual Saf 2017;26:33-41.

26. Hill AM, Etherton-Beer C, Haines TP. Tailored education for older patients to facilitate engagement in falls prevention strategies after hospital discharge--a pilot randomized controlled trial. PLoS One 2013;8:e63450

27. D-Ca L, Pritchard E, McDermott F, et al. Falls prevention education for older adults during and after hospitalization: A systematic review and meta-analysis. Health Educ J 2013;73:530-44.

28. Hill AM, Etherton-Beer C, McPhail SM, et al. Reducing falls after hospital discharge: a protocol for a randomised controlled trial evaluating an individualised multimodal falls education programme for older adults. BMJ Open 2017;7:e013931.

29. Michie S, van Stralen MM, West R. The behaviour change wheel: a new method for characterising and designing behaviour change interventions. Implement Sci 2011;6:42.
30. Creswell JW. Research design: qualitative, quantitative, and mixed methods approaches. 4th edn. Thousand Oaks: SAGE Publications, 2014.

31. Craig P, Dieppe P, Macintyre S, et al. Developing and evaluating complex interventions: the new Medical Research Council guidance. BMJ 2008;337:a1655.

32. Jones A, Bugge C. Improving understanding and rigour through triangulation: an exemplar based on patient participation in interaction. J Adv Nurs 2006;55:612-21.

33. Liamputtong PE. Research methods in health: foundations for evidencebased practice. 2nd edn. Victoria: Oxford University Press, 2013.

34. Hodkinson HM. Evaluation of a mental test score for assessment of mental impairment in the elderly. 1972. Age Ageing 2012;41(Suppl 3):iii35-iii40.

35. Katz S. Assessing self-maintenance: activities of daily living, mobility, and instrumental activities of daily living. J Am Geriatr Soc 1983;31:721-7

36. Lawton MP, Brody EM. Assessment of older people: selfmaintaining and instrumental activities of daily living. Gerontologist 1969;9:179-86.

37. Hill AM, McPhail SM, Waldron N, et al. Fall rates in hospital rehabilitation units after individualised patient and staff education programmes: a pragmatic, stepped-wedge, cluster-randomised controlled trial. Lancet 2015;385:2592-9.

38. Hartley J. Some thoughts on Likert-type scales. Int J Clin Health Psychol 2014;14:83-6.

39. Resnick B, Jenkins LS. Testing the reliability and validity of the selfefficacy for exercise scale. Nurs Res 2000;49:154-9.

40. Yesavage JA, Brink TL, Rose TL, et al. Development and validation of a geriatric depression screening scale: a preliminary report. $J$ Psychiatr Res 1982;17:37-49.

41. Richardson JR, Peacock SJ, Hawthorne G, et al. Construction of the descriptive system for the assessment of quality of life AQoL-6D utility instrument. Health Qual Life Outcomes 2012;10:38.

42. Braun V, Clark V. Successful qualitative research; a practical guide for beginners. London: Sage, 2014.

43. Creswell JW. Educational research: planning, conducting, and evaluating quantitative and qualitative research. 4th ed. Boston: Pearson, 2012.

44. StataCorp. Stata: release 14. statistical software. College Station, TX: StataCorp, 2013.

45. Gupta SK. Intention-to-treat concept: A review. Perspect Clin Res 2011:2:109-12.

46. Smith JA, Flowers P, Larkin M. Interpretative phenomenological analysis: theory, method and research. Los Angeles: SAGE, 2009.

47. Howard-Anderson J, Lonowski S, Vangala S, et al. Readmissions in the era of patient engagement. JAMA Intern Med 2014;174:1870-2.

48. Gonçalves-Bradley DC, Lannin NA, Clemson LM, et al. Discharge planning from hospital. Cochrane Database Syst Rev 2016.CD000313.

49. Michie S, Richardson M, Johnston M, et al. The behavior change technique taxonomy ( $v 1)$ of 93 hierarchically clustered techniques: building an international consensus for the reporting of behavior change interventions. Ann Behav Med 2013;46:81-95.

50. Abraham C, Wood CE, Johnston M, et al. Reliability of identification of behavior change techniques in intervention descriptions. Ann Behav Med 2015;49:885-900.

51. Michie S, Wood CE, Johnston M, et al. Behaviour change techniques: the development and evaluation of a taxonomic method for reporting and describing behaviour change interventions (a suite of five studies involving consensus methods, randomised controlled trials and analysis of qualitative data). Health Technol Assess 2015;19:1-188

52. Moore GF, Audrey S, Barker M, et al. Process evaluation of complex interventions: Medical Research Council guidance. BMJ 2015;350:h1258. 